\title{
Modelling of the rise and absorption of a fluid inclusion
}

\author{
Anatoliy Vorobev ${ }^{\mathrm{a}}$, Elena Khlebnikova ${ }^{\mathrm{b}}$ \\ ${ }^{a}$ Faculty of Engineering and the Environment, University of Southampton, Southampton, \\ SO17 1BJ, UK \\ ${ }^{b}$ Department of Fuel Engineering and Chemical Cybernetics, Institute of Natural Resources, \\ National Research Tomsk Polytechnic University, Tomsk, 632050, Russia
}

\begin{abstract}
The buoyancy-driven rise of a small single fluid inclusion in a viscous liquid that is enclosed in a container of the cylindrical shape is numerically modelled. It is additionally assumed that the inclusion is slowly absorbed by the surrounding liquid. The phase-field approach is used as a physics-based model for the description of the thermo- and hydrodynamic evolution of a miscible heterogeneous binary mixture. The interplay of the effects of absorption and inclusion's motion are investigated. We found that the dissolution of a nearly spherical inclusion does not occur evenly over its surface. The interfacial diffusion is stronger at the inclusion's top cap. The matter absorbed by the liquid does not quickly penetrate into the bulk of the ambient phase, it accumulates behind the inclusion forming a concentration wake. We found that during the rise the inclusion's speed grows. The speed and the acceleration of the inclusion strongly depend on the absorption rate, so the inclusion rises faster at the higher absorption rates. This effect is explained by the action of the Marangoni stress that is developed due to non-uniform mixture composition along the inclusion's surface. We also found that the rise of the inclusion in a closed container is accompanied by the recirculation flow that is developed near the inclusion and that rises upwards with the inclusion. In the limit of negligible absorption (higher Peclet numbers) the convergence to a constant terminal speed of an immiscible inclusion is observed.
\end{abstract}

Email address: A.Vorobev@soton.ac.uk (Anatoliy Vorobev)

Preprint submitted to Journal of ${ }^{A} T_{E} X$ Templates

April 18, 2018 
Keywords: miscible liquids, multiphase flows, diffusion, phase-field approach, rising bubble

\section{Introduction}

On the basis of the phase-field approach we model the evolution of a gaseous or liquid inclusion that rises in another liquid. It is additionally assumed that the inclusion is slowly absorbed by the ambient liquid, and the effects of the

5 absorption on the inclusion's speed, the inclusion's shape, and the effects of the inclusion's motion on the rates of absorption are investigated.

The evolution of a single inclusion is studied to comprehend the mixing dynamics of liquid/liquid and gaseous/liquid substances. The complete and fast mixing is a frequent pre-requirement for chemical interactions, and thus is highly desired in the majority of chemical engineering processes. In particular, it is known that slow and limited miscibility of gaseous species in liquids limits the reaction rates and the amounts of the reactants that partake in the process, forcing an extensive supply of the reactants, and thus leading to the problems of separation of products from non-reacted feedstock substances. To increase the area of contact of the phases, the reactants can be fed into a liquid-filled reactor in the form of small inclusions (the so called bubble column reactor).

A particular example would be the process of benzene alkylation that is used for production of ethylbenzene (a key intermediate for styrene production) with the yearly global demand exceeding 25 million tonnes [1, 2, Almost all ethylbenzene is synthesized from benzene and ethylene. In one of the variations of the process the alkylation is performed in the presence of mineral (Lewis) acids that are used as catalysts [1, 3, 4. These acids are highly toxic and corrosive and are frequently neutralised at the end of the reaction producing salts that need to be disposed. For the alkylation, benzene and catalyst are supplied in the liquid form, while ethylene is fed in the gaseous form as bubbles that rise in the reactor. Slow absorption of ethylene bubbles by the liquid phase limits the reaction rates, and intensification of the absorption can significantly 
reduce the usage of the reactants, including the catalyst, thus reducing the cost of the products and the environmental impact of the technology.

Another chemical engineering problem of the considerable interest and importance is the $\mathrm{CO}_{2}$ absorption by aqueous amine solutions that is considered as one of the most promising techniques for the $\mathrm{CO}_{2}$ capture [5, 6]. Optimisation and scale-up of this process is again significantly hampered by the low and slow miscibility of $\mathrm{CO}_{2}$.

Modelling of the dynamics of a single inclusion is the focus of the current study. The characteristics of a rising inclusion (its speed and shape) are affected by the different parameters that define the properties of both phases, such as density, viscosity, surface tension, etc. The hydrodynamic aspects of such motion have been thoroughly studied in numerous theoretical and experimental 40 works, see e.g. [7, 8, 9, 10, 11, 12, 13, 14, 15, 16, 17, 18, 19] and reviews [20, 21]. One of the current focuses of the similar studies is the development of new numerical models capable of accurate tracking of the liquid/bubble interfaces, as owing to a wide range of the available experimental data the rise of a single inclusion is considered as an excellent test for verification of the new numerical tools (see e.g. 222, 23, 24, 25, 26]). The phase-field approach was also proved to be an efficient method for the accurate modelling of complex shape transformations of a rising inclusion in a viscous liquid with large density and viscosity contrasts, see e.g. works [27, 28].

The rise of an inclusion that is absorbed by the ambient liquid was also examined in a number of experimental and numerical works. For instance, in [29], [18 the equation for the species balance is solved to determine the concentration fields in the ambient phase and thus to calculate the diffusion fluxes from a quasi-steady bubble (i.e. when all fields around the bubble can be regarded as steady) under assumption that the change of the bubble's shape (and size) due 55 to interfacial diffusion is negligible. The change of the bubble's size is taken into account in papers [30, 31, although under the assumption that the dissolved material is homogeneously spread in the surrounding liquid. This approach was further extended in paper 32 by taking into account the changes in the size 
of the bubble upon dissolution, although the bubble was assumed to remain spherical. In a recent work [33, the VOF method was modified by the addition of the source term, that takes into account the dissolution of the bubble, into the equation for the volume fraction function.

In the papers referred above the dissolution rate is defined as the difference between the concentration of the dissolved substances in the bubble's phase and the saturation level in the surrounding liquid. It should be also emphasised that the primary focus of these works is on the dissolution rates from a rising bubble, assuming that the dissolution is rather weak so its influence on the rising dynamics is neglected. Although we should mention that an addition of soluble surfactants changes the fluid velocity fields near rising bubbles, thus altering the speeds of bubbles' rise, see e.g. [34, 35, 20, 36].

In the current work we develop a new model that accurately describes the motion and the dissolution of the inclusion in a viscous liquid, fully resolving the flow fields within the inclusion and in the ambient phase, and tracing the transformations of the inclusion's shape due to mechanical action and due to dissolution/absorption [37. We also take into account the smearing of the interfacial boundary upon the dissolution, and the dynamic changes in the surface tension. The rate of interfacial diffusion is determined though the extended Fick's law, i.e. through the difference in the chemical potential, taking into account the effect of barodiffusion.

\section{Governing equations. Phase-field approach}

In the current work the phase-field approach is employed as a physics-based model capable of accurate description of the thermo- and hydrodynamic evolution of the binary mixture. The interface separating two liquids is represented as a transitional boundary of a finite thickness. The concentration field $C$, that is defined as the mass fraction of one component in the mixture, is used to trace the evolution of the interfacial boundary. The specific free energy function is 
defined as a function of concentration and concentration gradient 38,

$$
f(C, \nabla C)=f_{0}(C)+\frac{\epsilon}{2}(\nabla C)^{2} .
$$

Here the second term takes into account the surface tension effects. The role of this term is defined by the capillary constant $\epsilon$, which is assumed to be sufficiently small so to make these effects negligible everywhere save for the places of large concentration gradients, i.e. where interfaces are located.

The classical part of the free energy function, $f_{0}$, should define the possible states of the mixture. For instance, this part can be determined by the Landau formula,

$$
f_{0}(C)=a\left(C-C_{c r}\right)^{2}+b\left(C-C_{c r}\right)^{4} .
$$

This expression would be particularly suitable to represent the equilibrium states of binary mixtures with the so-called upper critical temperature (when the mixture is homogeneous in equilibrium above the critical temperature and may be heterogeneous below the critical temperature), especially, the states of the mixture in the vicinity of the consolute point (defined by the concentration $C_{c r}$ ). The phase diagram of such a mixture is defined by the equation, $\mu_{0}=\mathrm{d} f_{0} / \mathrm{d} C=0$, or, $\left(C-C_{c r}\right)^{2}=-a /(2 b)$. When $a /(2 b)$ is negative, the latter equation has two solutions,

$$
C=C_{c r} \pm\left(\frac{-a}{2 b}\right)^{1 / 2}
$$

that are associated with two components of the mixture. If $a /(2 b)$ is positive then the equation has only one homogeneous solution. Hence, the parameter $a /(2 b)$ can be interpreted as the difference of the temperature from the critical point $\left(T-T_{c r}\right)$.

We further assume that the behaviour of a mixture with the consolute point is examined. We however want to extend our consideration assuming that the binary mixture may occupy any physically possible thermodynamic state, that are not obligatory located near the critical point. The parameters $a$ and $b$ in our work are treated as the two phenomenological coefficients with the values to be 


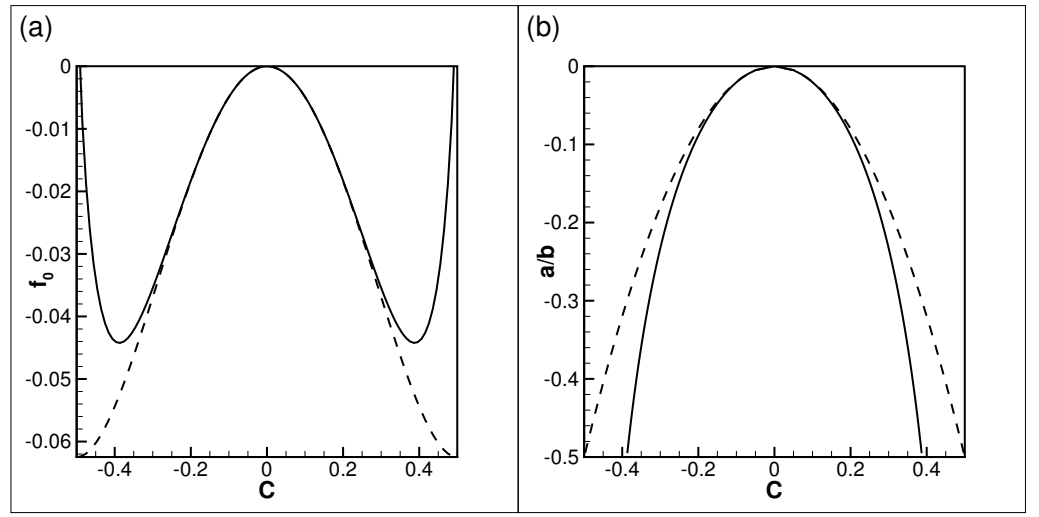

Figure 1: (a) The classical part of the free energy function (plotted for $a=-1 / 2$ and $b=1$ ), and (b) the phase diagram in the case of negligible surface tension effects. The solid lines depict the curves for the function (4) and the dashed lines depict the curves for the Landau function 2.

taken so to provide better matching with the experimental data. We however assume that $b$ remains positive while $a$ is either positive or negative depending on whether the binary system is single- or two-phase in equilibrium. In other words we extend the usual expectations allowed for the values of $a$ and $b$ near the consolute point to all possible thermodynamic states of the binary mixture.

For convenience, we shift the reference point for the concentration field as follows, $C \rightarrow\left(C-C_{c r}\right)$. For simplicity we also assume that the phase diagram is symmetrical with the critical concentration $C_{c r}=1 / 2$. The values of the new concentration then vary within the range $[-1 / 2 ; 1 / 2]$.

In the presence of strong gravity-induced gradients in the field of concentration, or in the presence of strong flows, expression 22 becomes less convenient, as in these cases the numerically calculated values of the concentration can be outside of the range of concentrations that correspond to the pure components 
$( \pm 1 / 2), 1$ In 39 , 40 the other free energy function is used,

$$
\begin{array}{r}
f_{0}=\left(a-\frac{3}{2} b\right) C^{2}+ \\
+\frac{3}{4} b\left[\left(\frac{1}{2}+C\right) \ln \left(\frac{1}{2}+C\right)+\left(\frac{1}{2}-C\right) \ln \left(\frac{1}{2}-C\right)\right] .
\end{array}
$$

The logarithmic terms of this function, that are written similarly to the regular solution model frequently used in the theory of polymers [41, limit the values of concentration to the range $[-1 / 2 ; 1 / 2]$. In the case the symmetrical phase diagram, with the consolute point at $C=0$ (or for the old reference point, at $C=1 / 2$ ), expression (4) coincides with the Landau formula in the vicinity of the consolute point (with the accuracy of a constant term that is insignificant, as it disappears when the expression for the chemical potential is derived). Expression (4) is used for the current work to define the classical part of the free energy function. Figure 1 provides an illustration of the differences in the shape of the free energy function and in the shape of the phase diagram that are derived on the bases of the functions (2) and (4).

The full free energy function (1) can be used to re-derive the Navier-Stokes equations for the mixture, that was done by Lowengrub and Truskinovsky [42. The resultant full Cahn-Hilliard-Navier-Stokes equations are however impractical for numerical treatment due to quasi-compressibility, that is, the dependence of the mixture density on concentration. The Boussinesq approximation of the full equations [43] is used in the current work to define the evolution of the rising and absorbing inclusion in the liquid.

The governing equations reflect the laws of conservation of momentum, 145 species, and mass,

$$
\begin{array}{r}
\frac{\partial \vec{u}}{\partial t}+(\vec{u} \cdot \nabla) \vec{u}=-\nabla \Pi+\frac{1}{\operatorname{Re}} \nabla^{2} \vec{u}-C \nabla \mu, \\
\frac{\partial C}{\partial t}+(\vec{u} \cdot \nabla) C=\frac{1}{\mathrm{Pe}} \nabla^{2} \mu,
\end{array}
$$

\footnotetext{
${ }^{1}$ The similar over-shootings are allowed by the shape of the free energy function 22, and such over-shootings are actually regularly observed in the numerical results obtained on the basis of the phase-field approach.
} 


$$
\begin{array}{r}
\nabla \cdot \vec{u}=0, \\
\mu=-\operatorname{Gr}(\vec{r} \cdot \vec{\gamma})+\mu_{0}-\mathrm{Cn}^{2} \nabla^{2} C, \\
\mu_{0}=\frac{d f_{0}}{d C}=\frac{3}{4} \ln \left(\frac{1 / 2+C}{1 / 2-C}\right)-(3-2 \mathrm{~A}) C .
\end{array}
$$

These equations are applied to the whole multiphase system, including the interface. Here, $\vec{u}$ is the velocity vector (defined as the mass averaged velocity for a fluid particle composed of two different components), $\Pi$ is the modified pressure field that is to be determined from the incomressibility constraint, and $150 \mu$ is the chemical potential ( $\mu_{0}$ is the classical part of the chemical potential). The Navier-Stokes equation contains an additional (Korteweg) force that determines the interface morphology and the hydrodynamic flows induced near the interface mimicking the Marangoni effect. The intensity of the diffusion process is defined by the extended Fick's law, i.e. through the gradient of the chemical potential $\mu$, which allows us to include, in addition to the usual diffusion driven by the concentration variations, the barodiffusion terms. The unit vector $\vec{\gamma}=-\vec{g} / g$ is opposite to the vector of the gravity acceleration.

The equations are written in non-dimensional form and include the following parameters. The Grashof number,

$$
\mathrm{Gr}=\phi \frac{g L_{*}}{\mu_{*}},
$$

the Reynolds number,

$$
\operatorname{Re}=\frac{\rho_{*} \mu_{*}^{1 / 2} L_{*}}{\eta_{*}}
$$

the Peclet number,

$$
\mathrm{Pe}=\frac{\rho_{*} L_{*}}{\alpha \mu_{*}^{1 / 2}},
$$

and the Cahn number,

$$
\mathrm{Cn}=\left(\frac{\epsilon}{\mu_{*} L_{*}^{2}}\right)^{1 / 2} .
$$

In these formulae, $L_{*}$ is the typical size, $\rho_{*}$ is used as the density scale, $\mu_{*}$ is the unit of the chemical potential, $\eta_{*}$ is the viscosity scale, $\alpha$ is the mobility 165 
the densities of the pure components of the binary mixture. The typical scale of the velocity field is $\mu_{*}^{1 / 2}$.

The parameter $\mathrm{A}=a / b$ comes from the equation for the free energy function (4), and this parameter determines the thermodynamic states of the mixture.

It is necessary to note that the above definitions of the non-dimensional parameters are different from the standard definitions that would be given on the basis of their standard phenomenological parameters, such as the surface tension and diffusion coefficients. We keep using the conventional names and notations, as these non-dimensional parameters appear in front of the corresponding terms of the hydrodynamic equations. The Cahn number, $\mathrm{Cn}$, is also referred to as the capillary number as its value determines the importance of the capillary effects. It can be also shown that this parameter is proportional to the thickness of an equilibrium interface between two phases.

It is also necessary to note that the governing equations (5) were derived for two liquids with different viscosities [43. In the derivation, this difference was assumed small, and thus only one single Reynolds number was introduced. Although, for the case when the viscosity difference is large, the governing equations need to be reconsidered, which may bring several new terms. This has not been done in the present work.

185 It can be shown that the surface tension coefficient associated with the phase boundary can be defined as

$$
\sigma_{\infty}=\mathrm{Cn}^{2} \int_{-\infty}^{\infty}\left(\frac{d C}{d x}\right)^{2} d x
$$

where $x$ is the coordinate across the interface. For a flat equilibrium interface and with no gravity effect, the interface thickness can be evaluated as $\delta_{e q}=$ $\mathrm{Cn} / \sqrt{-\mathrm{A}}$ and the surface tension coefficient as $\sigma_{\infty}=\frac{1}{3} \mathrm{Cn} \sqrt{-\mathrm{A}}$ (see e.g. [44]).

In addition, it is necessary to note that on a macroscopic scale the interface between two liquids is infinitely sharp, and hence the limiting behaviour of the multiphase system for $\delta \rightarrow 0$ is of the primary interest for the phase-field approach. 


\section{Problem statement and numerical approach}

195 is represented by a vertical cylinder with circular cross section. The cylinder's radius is used as the length scale. The radial and axial coordinates are denoted by $r$ and $z$, respectively. The cylinder is closed at the bottom and top ends. The inclusion rises along the centreline. The axial symmetry is assumed, and

equilibrium value $\delta_{e q}$. This is one of the main differences of the current work from other similar studies (see e.g. [27, 28]), where the phase-field method

\footnotetext{
${ }^{2}$ We examine the motion of the inclusions with the radii that are lower than 0.3 . The influence of the walls on the rise of similar inclusions is not negligible, although it remains sufficiently low. The detailed study of the influence of the pipe walls on the motion of a single immiscible bubble is reported in work [8].
} 
is used for modelling the evolution of a binary system that experiences some hydrodynamic changes, assuming although that the system either resides in a state of thermodynamic equilibrium (i.e. $\delta_{0}=\delta_{e q}$ ) or very near the equilibrium $\left(\delta_{0} \approx \delta_{e q}\right)$ and thus the interfacial diffusion is negligible. We use the phase-field approach as a physics-based model for miscible heterogeneous systems that is capable of tracing the transformations of the inclusion's shape and interfacial diffusion through the interface. The focus of works [27, 28, was to model the hydrodynamic evolution of a bubble with exclusion of the diffusion effects.

The governing equations (5) are supplemented with the following boundary conditions. At the lower end,

$$
z=0: u_{r}=u_{z}=0, \frac{\partial C}{\partial z}=0, \frac{\partial \mu}{\partial z}=0 .
$$

At the upper end,

$$
z=H: u_{r}=u_{z}=0, \frac{\partial C}{\partial z}=0, \frac{\partial \mu}{\partial z}=0 .
$$

At the centreline,

$$
r=0: u_{r}=0, \frac{\partial u_{z}}{\partial r}=0, \frac{\partial C}{\partial r}=0, \frac{\partial \mu}{\partial r}=0 .
$$
inclusion far from the walls, so the wetting properties are not important for the current study. The boundary conditions at the centreline are written to reflect the axial symmetry. 
For the numerical solution, the equations are re-written into the streamfunctionvorticity formulation. The resultant equations are solved using the finite-difference method on a uniform mesh. The explicit first order in time and second-order in space discretisation scheme is used.

To characterise the evolution of the inclusion we calculate a number of the integral parameters. Namely, we determine the vertical position of the inclusion, $z_{d}$, and the speed of the inclusion's rise, $U=\mathrm{d} z_{d} / \mathrm{d} t$. The rise of the inclusion induces the hydrodynamic motion in the ambient phase, and the intensity of the motion is determined by the total kinetic energy, $E_{k}=\int_{V} u^{2} \mathrm{~d} V$ (here $V$ is the total volume of the computational domain). We also calculate the inclusion's volume, $V_{d}$ (defined as the volume of the region for which $C<0$ ), the volume of the transitional layer between the phases, $V_{\delta}$ (the volume of the zone for which $|C|<0.05$ ), the surface area of the inclusion, $S$ (for the axisymmetric problem the task of finding this quantity is reduced to the calculation of the length of the line in the $x-z$ plane defined by the condition $C=0$ ), the thickness of the transitional zone, $\delta=V_{\delta} / S$, and the surface tension coefficient,

$$
\sigma=\frac{\mathrm{Cn}^{2}}{S} \int_{V}\left(\left(\frac{\partial C}{\partial r}\right)^{2}+\left(\frac{\partial C}{\partial z}\right)^{2}\right) \mathrm{d} V
$$

We characterise the changes of the inclusion's shape by determining its two dimensions, $a_{r}$ and $a_{z}$, which are the maximum sizes of the inclusion in the radial and vertical directions. We also calculate the average concentrations in each phase.

Figure 2 shows the time evolutions of the integral quantities for one of the runs that is chosen here for the illustration of the numerical convergence of the results. In figure 2 one clearly see that the differences between the lines are gradually reduced when the number of the grid nodes is increased. The most of the results of this work are obtained with the use of the numerical grids with either $350 \times 2100$ or $400 \times 2400$ nodes. The improved resolution $(450 \times 2700)$ is used for some calculations that are performed for higher Peclet numbers, when the use of the finer numerical grid gives visible improvements. The need of the improved resolution at higher Peclet numbers is to be explained by the smaller 

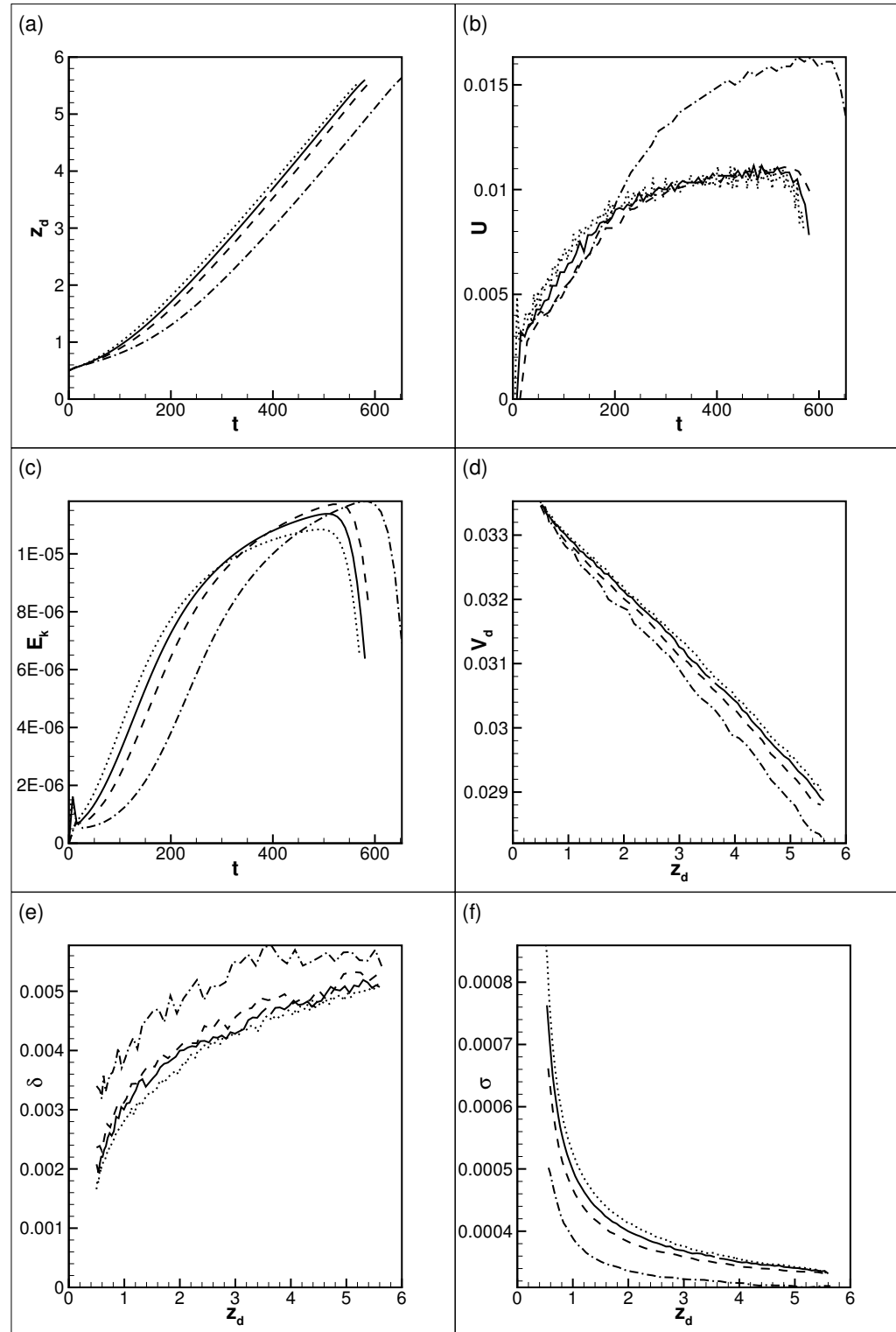

Figure 2: (a) The vertical position of the inclusion, (b) the speed of the inclusion's rise, and (c) the total kinetic energy of the fluid flow induced in the computational domain vs time. (d) The volume of the inclusion, (e) the thickness of the interfacial boundary, and (f) the surface tension coefficient vs. the vertical position of the inclusion. The results are obtained for $H=6, \mathrm{~A}=-0.5, r_{0}=0.2, \mathrm{Pe}=5 \cdot 10^{6}, \mathrm{Gr}=0.01, \mathrm{Re}=100, \mathrm{Cn}=0.005$, and with the numerical grids with $250 \times 1500$ nodes (dash-dotted lines), $350 \times 2100$ (dashed lines), $400 \times 2400$ (solid lines), and $450 \times 2700$ (dotted lines). 
diffusive length scales $3^{3}$ and by the weaker diffusive damping of the numerical instabilities (in 44, 45, 46], it is shown that the diffusion effect damps the growth of physical and numerical instabilities similar to the effect of viscosity).

In figure 2 one sees that the chosen resolution is sufficient for tracing the rise of the inclusion, although, it is not sufficient to get rid of all fluctuations in some of the curves. For instance, the initial thickness of the interface is equal to 4 grid steps (which is considered as the minimum number of the grid points across the interface that is needed for the accurate interface tracking with the use of the phase-field approach, see e.g. [27, 40]), and since the minimum increment to the interface thickness is equal to one grid point, i.e. comparable with the size of the interface, these increments are well visible in figure 24 As can be seen in figure 280 , for the chosen set of parameters the overall changes of the inclusion's volume are relatively small, and hence some fluctuations on these curves are also visible. The changes of the vertical coordinate of the inclusion are not that small, so the lines in figure $2 \mathrm{a}$ are smooth. Nevertheless, the inclusion rises slowly. We calculate the inclusion's speed by taking the discrete positions of the inclusion separated by the time step of 4 (in non-dimensional time units). Owing to the relatively small values of the resultant inclusion's speed the instantaneous fluctuations on these curves (figure $2 \mathrm{~b}$ ) are also visible. These fluctuations are of the purely numerical nature, and they do not affect the general dependencies for the quantities shown in figure 2 and in the other figures of this paper. 


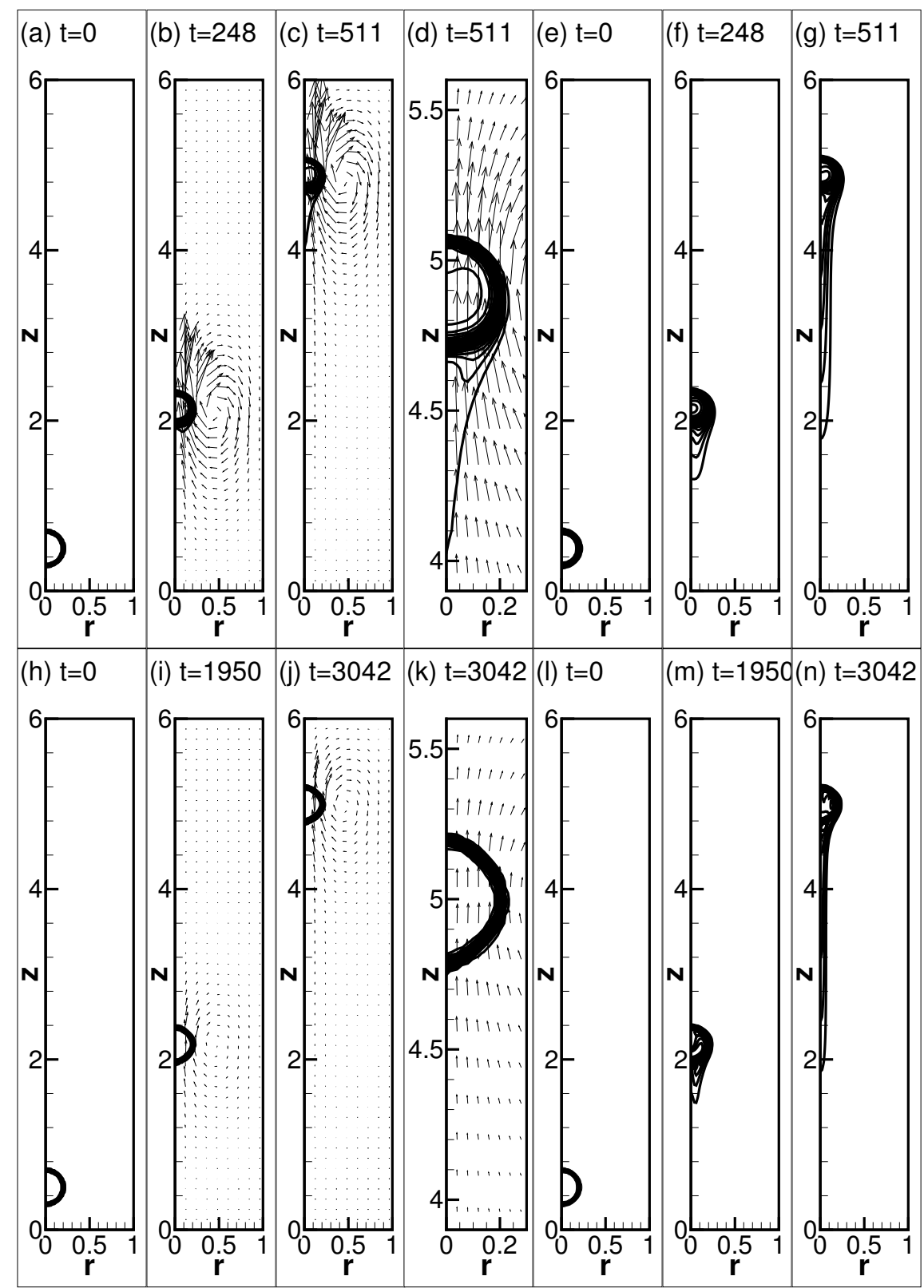

Figure 3: The snapshots showing the stages of the rising bubbles with the initial radius of $r_{0}=0.2$ at two Peclet numbers, $\mathrm{Pe}=5 \cdot 10^{6}(\mathrm{a}-\mathrm{g})$ and $\mathrm{Pe}=1.6 \cdot 10^{8}(\mathrm{~h}-\mathrm{n})$. The isolines represent the fields of concentration (a-d, h-k) and chemical potential (e-g, l-n), and the vectors depict the velocity fields (with the lengths scaled in accordance with the velocity magnitude). The results are obtained for $H=6, \mathrm{~A}=-0.5, \mathrm{Gr}=0.01, \mathrm{Re}=100, \mathrm{Cn}=0.005$. The actual time moments are depicted in the figure. (d) and (k) are the enlarged versions of (c) and (j). 


\section{Results}

Figure 3 shows the fields of concentration, chemical potential, and velocity at different time moments. These snapshots are obtained from the numerical runs fulfilled for two different Peclet numbers. The inclusion has a spherical shape and zero velocity at the initial moment. By the action of the gravity force the inclusion starts rising, although its speed seems to be rather low, as its shape remains nearly spherical. In figure 3 the results are shown for two different Peclet numbers (with the stronger and weaker diffusion effects), and one can clearly see that the speed of the inclusion, and the structure of the fields of the concentration and chemical potential are strongly affected by the

300 the inclusions shown in figure 4 rise very slowly, the vortex flow should not be associated with the wake flow that appears behind the quickly rising bubbles

\footnotetext{
${ }^{3}$ At the start of the inclusion's rise, when the time changes are determined by the hydrodynamic motion, the diffusive length scale can be estimated as $l^{2} \sim \operatorname{Re} / \mathrm{Pe}$.

${ }^{4}$ The initial interface thickness is different for the calculations conducted with the different resolutions, as can be seen in figure 2 This can easily explained by the fact that the initial interface thickness was set as 4 grid steps and thus it depends on the grid size. This difference also explains the different values of the surface tension (that is reciprocal to the interface thickness). These differences although have no effects on the values of the other integral parameters.
} 
20, 18. In the current work the rise of the inclusion in a closed container is considered, and thus the upward motion of an incompressible fluid along the centerline needs to be accompanied by a descending recirculation flow along the container's walls. One however sees that this recirculation is achieved by the vortex flow that does not occupy the entire cylinder. The visible hydrodynamic motion is bound to the zone near the inclusion, and although the vortex' size exceeds the size of the inclusion, these two quantities remain of the same order. The vortex is attached to the inclusion over its entire rise. We performed the numerical simulations for the longer cylinders, with the heights up to $H=$ 12, and all observations coincide with those reported here up to the moment the inclusion's vertical coordinate is below 5.5, i.e. while the influence of the upper boundary is negligible, which allows us to conclude that the flow pattern observed in figure 3 is pertinent to the rise of the inclusion in a closed cylinder, and, e.g., it does not depend on the cylinder's height.

It is interesting that at higher Peclet numbers the inclusion rises slower, and the rise of the inclusion induces much weaker hydrodynamic motion in the ambient phase. The strong dependence of the ascending speed on the Peclet number should be related to the time changes of the species distribution. A possible mechanism that may induce the flow in the surrounding liquid is the solutal convection that may be generated by the concentration inhomgeneities. Although, even for the case with stronger diffusion effect, the typical diffusive time remains much longer than the typical convective time. In figure 4 one sees that the inhomgeneities in the concentration field only develop within the inclusion and behind the inclusion. At the inclusion's side the concentration field remains homogeneous, and hence the convective mechanism may not explain the observed flows, and may not explain the observed dependencies on the Peclet number.

340 The complex time variations of the ascending velocity of the bubbles was earlier observed for the bubbles rising in the liquid with added surfactants that either are absorbed or desorbed by the bubbles [34, 35, 20, 36. In e.g. [35, it is reported the speed of a bubble rising in the surfactant-rich solution in- 
creases at the initial moment, reaches some maximum value and then slowly decreases to the terminal velocity that is different from the terminal velocity of a bubble rising in pure water. The observed dependencies are explained by the slow absorption of the surfactant by the bubble surface, and, consequently, by the Marangoni forces induced at the surface due to uneven distribution of the surfactants along the bubble's surface. These Marangoni forces introduce the additional drag force that slows down the rise of the bubble.

The strong dependence of the inclusion on the Peclet number that is observed in figure 4 should be explained by the same mechanism. The Korteweg term in the Navier-Stokes equation (5) generates the additional force, that, in our case, assists the rise of the inclusion, increasing its ascending speed. The faster inclusion's rise results in the faster recirculation flow.

In figure 3 one can also notice that the dissolution of the matter primarily occurs at the inclusion's top cap (where the gradient of the chemical potential has the greater values). The dissolved matter is then transported by the vortex to the inclusion's rear side, where the tales in the fields of concentration and chemical potential are formed. The formation of the similar concentration tail (or wake) that accumulates the dissolved matter was also reported in the numerical studies [32, 33]. The advection of the surfactant along the bubble surface and then accumulation of the surfactant in the rear section of the bubble was also reported in works [34, 20, 36].

The time evolutions of the integral parameters that characterise the rise of the inclusion are depicted in figure 4. Figure 4 a depicts the vertical position of the inclusion at different time moments and figure $4 \mathrm{~b}$ depicts the inclusion's speed versus time. One sees that the inclusion starts to accelerate at $t=0$, and the inclusion's speed never becomes constant increasing over the entire rise. Nevertheless, the speed itself and the rate of its changes are lower at the higher values of the Peclet number.

The rise of the inclusion induces the hydrodynamic motion in the computational domain. The intensity of the generated hydrodynamic motion keeps growing over the entire rise of the inclusion, although, the kinetic energy itself 

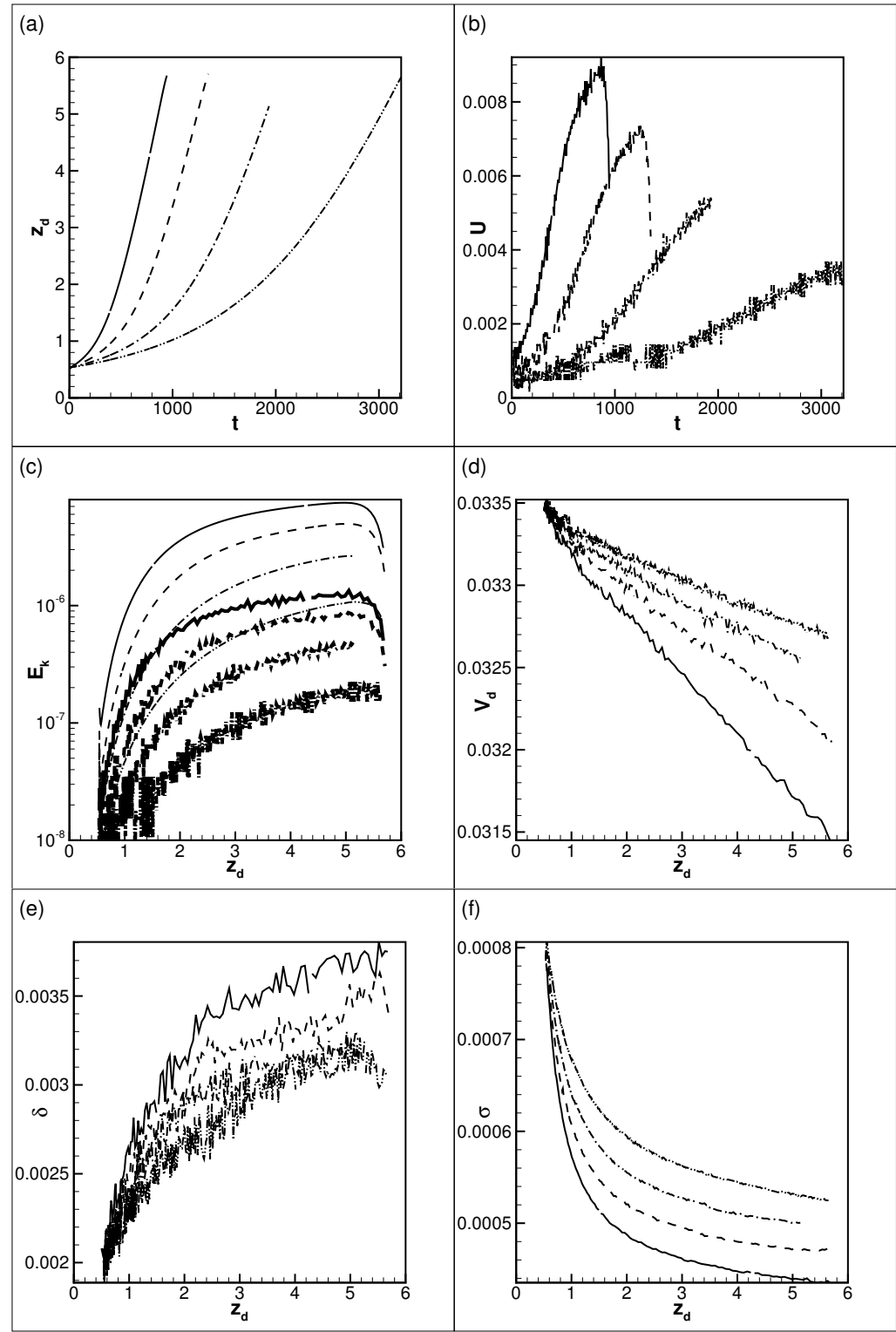

Figure 4: (a) The vertical position of the inclusion, (b) the speed of the inclusion, (c) the total kinetic energy of the binary mixture and the kinetic energy of the inclusion (thick lines) vs. time. (d) The volume of the inclusion, (e) the thickness of the interface, (f) the surface tension vs. the inclusion's vertical position. The data is plotted for $r_{0}=0.2, H=6, \mathrm{~A}=-0.5$, $\mathrm{Gr}=0.01, \mathrm{Re}=100, \mathrm{Cn}=0.005$, and $\mathrm{Pe}=2 \cdot 10^{7}$ (solid lines), $\mathrm{Pe}=4 \cdot 10^{7}$ (dashed lines), $\mathrm{Pe}=8 \cdot 10^{7}$ (dash-dotted lines), and $\mathrm{Pe}=1.6 \cdot 10^{8}$ (dash-dot-dotted lines). 


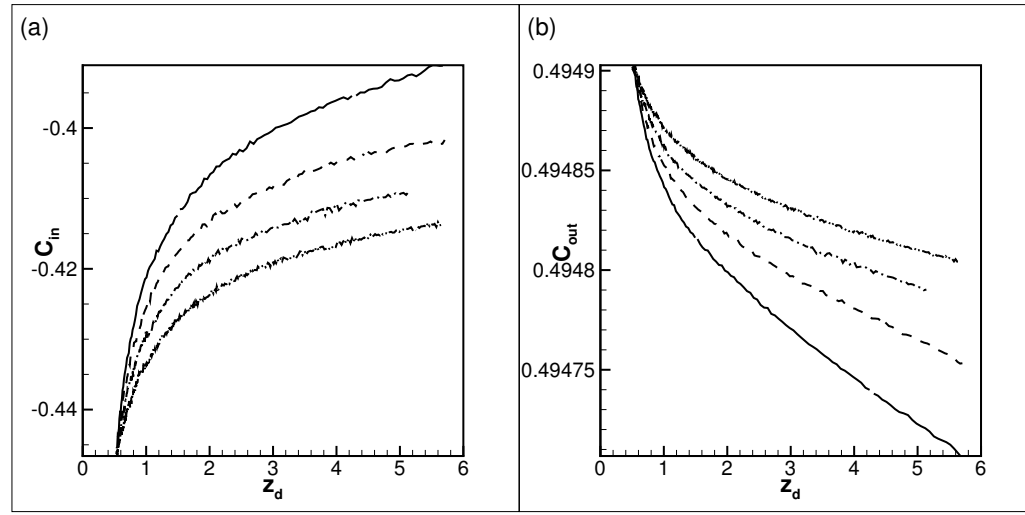

Figure 5: The dependences of the average concentrations in the inclusion (a) and in the ambient liquid (b) phases on the vertical coordinate of the inclusion. The data are plotted for the same parameters as in figure 4

375 and its time changes become smaller at higher Peclet numbers (figure 4k). In figure 4k, the kinetic energy that is solely associated with the inclusion, that is determined as $V_{d} U^{2} / 2$, is also shown by using the thicker lines. One can see that this part in the total kinetic energy of the system is at least by an order lower than the total kinetic energy, indicating that the volume of the liquid that is involved into the hydrodynamic motion is in fact larger than just the inclusion's volume.

Figure 4 $4 \mathrm{~d}$ illustrates the decrease of the inclusion's volume during the rise. The volume decreases linearly in respect with the inclusion's vertical coordinate. The rate of the volume's decrease obviously depends on the Peclet number, being higher at lower Peclet numbers. Owing to diffusion the inclusion's interface smears but this happens very slowly as seen in figure 44. Following the slow interface smearing the surface tension associated with the inclusion's interface slightly decreases. The rate of the interface smearing and, consequently, the rate of the decay of the value of the surface tension coefficient are slightly weaker at the higher Peclet numbers.

Finally, in figure 5 we show the time dependences of the average concentrations within the inclusion and within the ambient liquid. One sees that the 
shrinkage of the inclusion (figure $4 \mathrm{~d}$ ) and the smearing of the interface (figure 4) is driven by the exchange of the molecules between the phases. The dissoluthe ambient phase (figure $5 \mathrm{~b}$ ), although, the dissolution of a smaller inclusion in a much larger computational domain has a relatively small impact on the value of the average concentration. For the inclusion phase, the relative changes in the average concentration are much more pronounced (figure 5 a), and here one could observe the adjustment of the average concentration to the value -0.388 that is the equilibrium concentration for $A=-1 / 2$ (see also figure 1 that defines the phase diagram).

Figures 6 and 7 depict the data that characterise the inclusion when it reaches the position with the vertical coordinate $z=5$. At this moment, the inclusion keeps accelerating, and thus the speeds shown in figures $6 a, b$ and $7 a, b$ do not correspond to the terminal speeds that are usually used to characterise the rise of the inclusion. Nevertheless, as it was earlier mentioned the rate of changes of the inclusion's speed are lower for the higher Peclet numbers. For a smaller inclusion (with the initial size, $r_{0}=0.1$ ) we managed to fulfil the numerical runs for the sufficiently high Peclet numbers $\left(\mathrm{Pe}=3.2 \cdot 10^{8}\right)$. The results show that the curves of the inclusion's speed clearly converge to the values that can determine the rise of immiscible inclusions, given by the Hadamard-Rybczynski equation, i.e.

$$
U_{\infty}=\frac{4}{15 K_{1}} \operatorname{GrRe}_{0}^{2} .
$$

The latter formula determines the terminal speed of a small spherical immiscible inclusion, assuming that the components a binary mixture have a small difference in viscosity coefficients [47]. This expression is written in the nondimensional form using the scales and the non-dimensional parameters introduced above. The coefficient $K_{1}$ stands for the correction factor that takes into account the additional drag enforced on the inclusion's motion by the walls of 420 the cylinder (i.e. by the boundaries of the computational domain) [8]. This correction factor is a strong function of the inclusion's size, and for the inclu- 

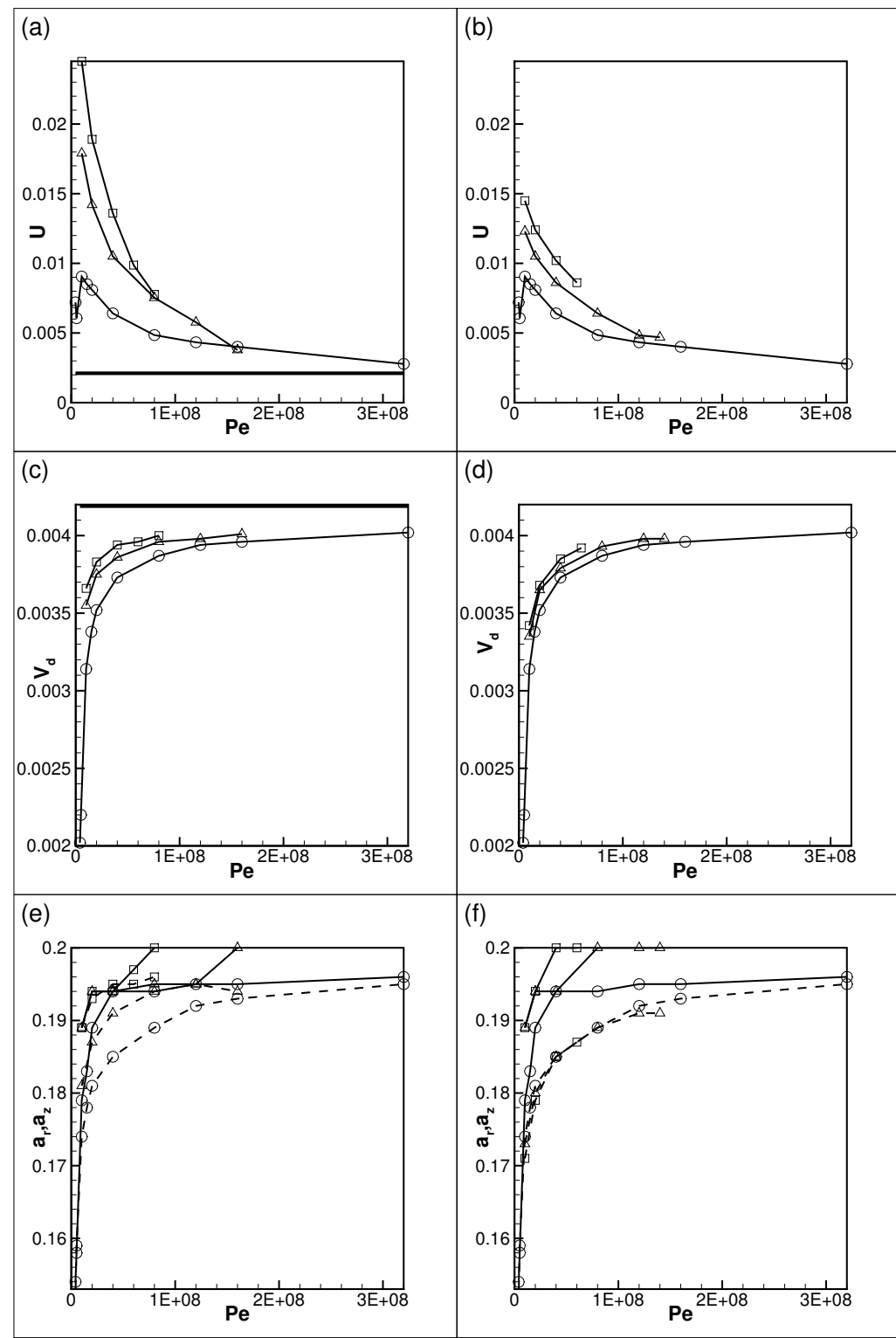

Figure 6: $(\mathrm{a}, \mathrm{b})$ The speed of the inclusion at $z=5,(\mathrm{c}, \mathrm{d})$ the volume of the inclusion at $z=5$, and (e,f) the horizontal ( $a_{r}$, solid lines) and vertical $\left(a_{z}\right.$, dashed lines) sizes of the inclusion at $z=5$ vs. Peclet number. The data are plotted for $H=6, \mathrm{~A}=-0.5, r_{0}=0.1, \mathrm{Cn}=0.005$, and (a,c,e) for $\mathrm{Gr}=0.01$ and three Reynolds numbers $\operatorname{Re}=100$ (circles), $\operatorname{Re}=200$ (triangles), and $\operatorname{Re}=300$ (square symbols), (b,d,f) for $\mathrm{Re}=100$, and three Grashof numbers $\mathrm{Gr}=0.01$ (circles), $\mathrm{Gr}=0.02$ (triangles), and $\mathrm{Gr}=0.03$ (square symbols). The horizontal vertical line in (a) depicts the terminal velocity determined from formula 21 for $R e=100$, and the horizontal line in (c) depicts the initial volume of the inclusion. 

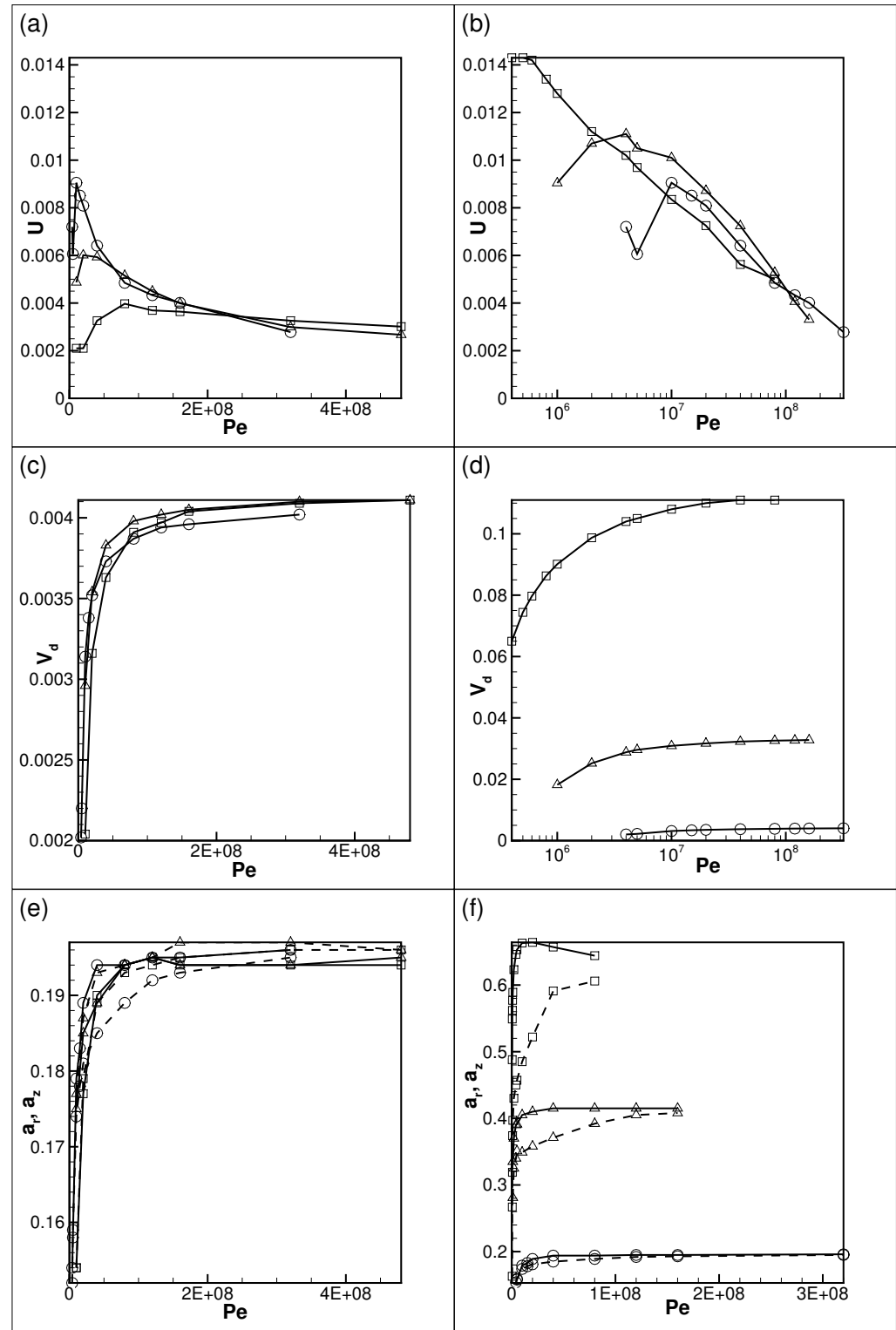

Figure 7: $(\mathrm{a}, \mathrm{b})$ The speed of the inclusion at $z=5,(\mathrm{c}, \mathrm{d})$ the volume of the inclusion at $z=5$, and $(\mathrm{e}, \mathrm{f})$ the horizontal $\left(a_{r}\right.$, solid lines) and vertical $\left(a_{z}\right.$, dashed lines $)$ dimensions of the inclusion at $z=5$ vs. Peclet number. The data are plotted for $H=6, \mathrm{~A}=-0.5$, $\mathrm{Re}=100, \mathrm{Gr}=0.01$, and $(\mathrm{a}, \mathrm{c}, \mathrm{e})$ for for $r_{0}=0.1$ and three Cahn numbers $\mathrm{Cn}=0.005$ (circles), $\mathrm{Cn}=0.01$ (triangles), and $\mathrm{Cn}=0.02$ (square symbols); and (b,d,f) for $\mathrm{Cn}=0.005$ and three initial radii of the inclusion, $r_{0}=0.1$ (circles), $r_{0}=0.2$ (triangles), and $r_{0}=0.3$ (squares). 
sions studied in this work, the values of the factor are 1.26 for $r_{0}=0.1,1.68$ for $r_{0}=0.2$, and 2.36 for $r_{0}=0.3$.

We demonstrate the convergence of the results for one set of the governing parameters, when the simulations for the sufficiently high Peclet number turned out to be feasible. For the other sets of the parameters, we have not reached the values set by formula (21), although the obtained values of the inclusion's speeds remain sufficiently close for the expected values. It needs to be emphasized that the inclusion's speeds shown in figures 6 and 7 correspond to the stage when the inclusion keeps accelerating and thus the motion of the inclusion is affected by the added-mass drag [47, 20, that is not taken into account by formula (21). The inclusion rise is also affected by the recirculation flow and by the Korteweg force. For smaller droplets and weaker interfacial diffusion these additional drag mechanisms are weaker, and thus some correspondence with equation 21) could be established. The strength of the Korteweg force is strongly dependent on the Peclet number and the added-mass drag and the drag imposed by the recirculation effect strongly depend on the inclusion size, resulting in the alteration of the standard dependences for the speed of the inclusion's rise on the governing parameters as they may be expected from equation (21).

For instance, figure 6 shows the data for the three different Reynolds numbers and for three different Grashof numbers, and figures $7 \mathrm{~b}, \mathrm{~d}, \mathrm{f}$ show the data for the three inclusions with different initial sizes. At lower Peclet numbers the speed of the inclusion's rise increases with the growth of the Reynolds and Grashof numbers and with the growth of the initial size of the inclusion, although not at the rate predicted by equation (21). At higher Peclet numbers the dependences on the Reynolds and Grashof numbers, and on the inclusion's size become even weaker.

In figures $6 \mathrm{a}, \mathrm{b}$ and $7 \mathrm{a}, \mathrm{b}$ one sees that the inclusion's speed strongly depends on the Peclet number, so that the inclusion rises faster at the stronger rates of absorption by the ambient phase (for the lower Peclet numbers). This observation remains valid until the absorption rate is so strong that the inclusion starts 
to lose the considerable portion of its initial volume.

Figures 7 a,c,e show the data obtained for the three different Cahn numbers. We observed that the inclusions that are being dissolved do not rise with the constant speed, and the aspect ratio and the sizes of the inclusion change due to dissolution. We may introduce the Reynolds number for an equivalent immiscible inclusion, with the velocity given by formula (21),

$$
\operatorname{Re}_{\infty}=\frac{8}{15} G r \operatorname{Re}^{2} r_{0}^{3},
$$


or we can introduce the actual Reynolds number,

$$
\operatorname{Re}_{U}=\operatorname{Re} U d
$$

that varies over the inclusion's rise and that is equal to $\operatorname{Re}_{\infty}$ in the limit of an immiscible inclusion. Here $d$ is the equivalent inclusion's diameter that can be defined as $d=\left(6 V_{d} / \pi\right)^{1 / 3}$.

From the obtained data we see that both $\operatorname{Re}_{\infty}$ and $\operatorname{Re}_{U}$ are in the range $[0.1 ; 1]$, which additionally confirms that the rise of a creeping Stokesian inclusion is studied in this work.

The mechanical deformation of the inclusion's shape during the rise is frequently characterised by the Morton, Eotvos (or Bond), and Weber numbers, which, in terms of the parameters introduced in this paper, are defined as

$$
\mathrm{Mo}=\frac{\mathrm{Gr}}{\operatorname{Re}^{4} \sigma^{3}}, \mathrm{Eo}=\frac{\mathrm{Gr} d^{2}}{\sigma}, \mathrm{We}=\frac{U^{2} d}{\sigma} .
$$

The surface tension and the speed of the inclusion are the functions of time in our work, and hence parameters (24) vary over the inclusion's rise. The estimations on the basis of the parameters of the current paper show that Mo $\sim 1$, Eo $\sim 1$, and We $\sim 0.1$, indicating that the inclusion could demonstrate some smaller shape deformations (see e.g. [15, 19]), obviously due low levels of the surface tension.

The dissolution process is also frequently characterised by the Schmidt number, that is $\mathrm{Sc}=\mathrm{Pe} / \mathrm{Re}$. This parameter varies in the range $\left[10^{4} ; 10^{6}\right]$.

Figures 8 a,c depict the aspect ratio of the inclusion against the Peclet and Weber numbers. The aspect ratio is small, approaches 1 at higher Peclet numbers, when the limit of an immiscible inclusion is expected. The deformation of the inclusion quickly grows at lower Peclet numbers, when the effect of dissolution is stronger and the speed of the inclusion's rise is also considerably higher.

The formula that determines the smaller deformations of inclusions for the case of small Weber numbers is reported in the work [10],

$$
\frac{a_{r}}{a_{z}}=1+0.337 W e
$$



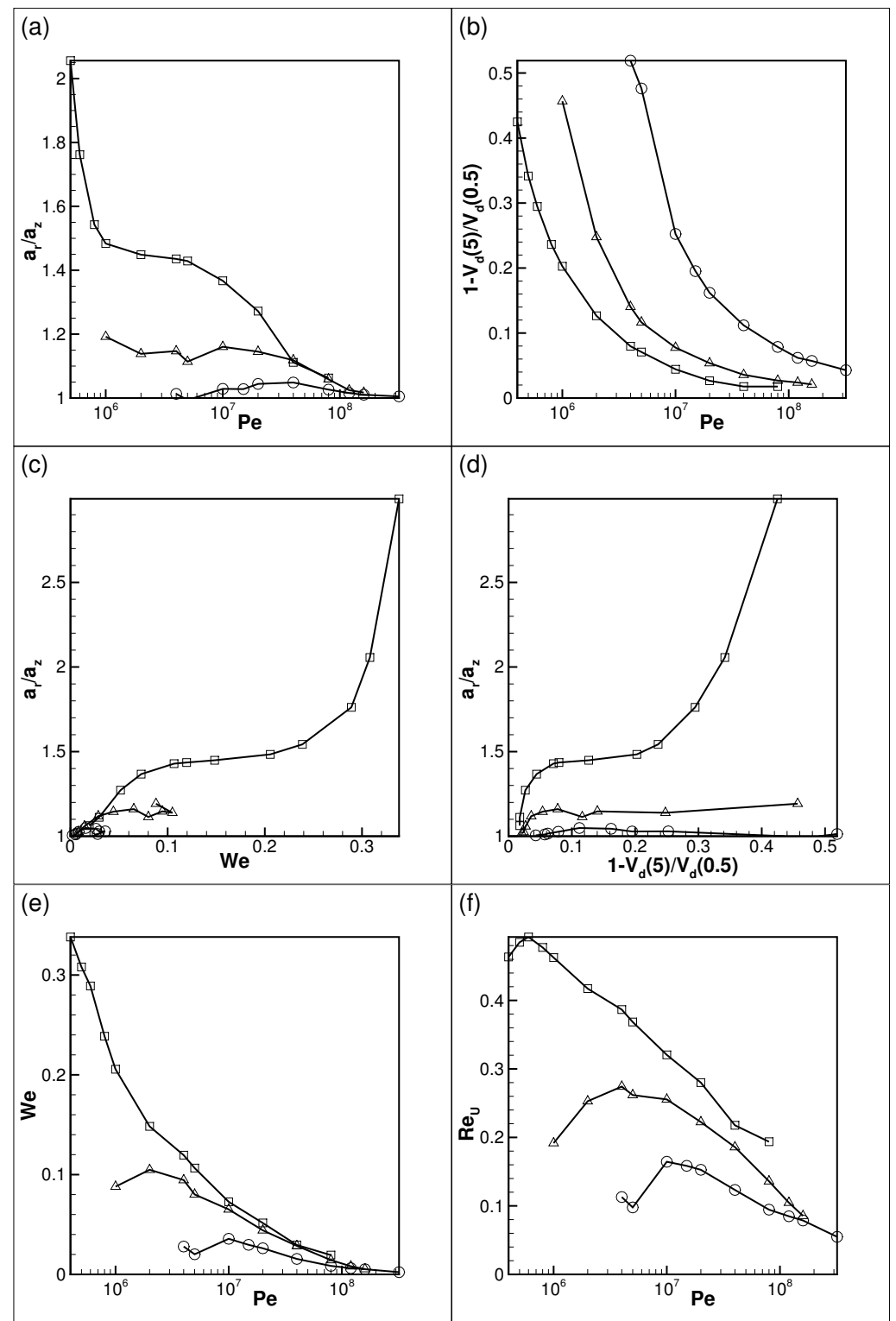

Figure 8: (a) The aspect ratio for the inclusion at $z=5$ vs. the Peclet number. (b) The relative changes of the inclusion's volumes between the initial point and the point $z_{d}=5 \mathrm{vs}$. Peclet number. (c,d) The aspect ratio vs. the Weber number and the relative changes of the inclusion's volumes (at $z=5$ ). (e,f) The Weber and Reynolds numbers for the inclusion calculated at $z=5$ vs. the Peclet number. The data are plotted for $H=6, \mathrm{~A}=-0.5$, $\mathrm{Cn}=0.005, \mathrm{Re}=100, \mathrm{Gr}=0.01$, and three different initial radii of the inclusion, $r_{0}=0.1$ (circles), $r_{0}=0.2$ (triangles), and $r_{0}=0.3$ (square symbols). 
We do not observe a similar dependence in the data depicted in figure 8 c, which indicates that the deformations of the inclusions reported in our work are primarily determined by the dissolution, so the much smaller mechanical deformations remain completely shadowed.

Figure $8 \mathrm{~b}$ shows that the relative changes of the inclusion volume. These changes are getting lower at higher Peclet numbers, which is obvious. What is more interesting is that the smaller inclusions are getting absorbed by the ambient liquid faster, in the sense that the relative changes of the volumes of smaller inclusions are greater. Figure $8 \mathrm{~d}$ depicts the dependences of the inclusion's aspect ratio against the relative change of the inclusion's volume. This figure clearly confirms our earlier conclusion that the variations in the inclusion's shape should be related to uneven dissolution from the inclusion's surface, rather to mechanical deformations. The dependencies of the Weber number and Reynolds number, $\mathrm{Re}_{\mathrm{U}}$, on the Peclet number depicted by figures ${ }_{520} 8$; ,e are also fully explained by the strong dependence of the inclusion's speed on the Peclet number.

In the end of the work we would like to provide some correlation of the observations of this work to a real system. The phase-field method is based on the use of some non-standard phenomenological parameters, such as the 525 capillary and mobility constants, as well as the scale for the chemical potential. The values of these parameters can be determined indirectly by comparison of the numerical and experimental results (see e.g. 40]). The main parameter is the typical value of the chemical potential, $\mu_{*}$, since it enters all governing parameters that define our mathematical model. The capillary and mobility constants are then can be estimated from the values of the capillary and Peclet numbers.

Let us assume that a tiny inclusion with the typical size of $0.1 \mathrm{~mm}$ (hence $L_{*}$, which is the radius of the computational domain, is $1 \mathrm{~mm}$ ) rises in a liquid with a typical density $\rho_{*} \sim 10^{3} \mathrm{~kg} / \mathrm{m}^{3}$ and typical viscosity coefficient $\eta_{*} \sim 10^{-3} \mathrm{~Pa} \cdot \mathrm{s}$.

Our main results are obtained for $\mathrm{Re}=100$. Using the definition of the Reynolds number (11), we may derive the typical value of the chemical potential 
for a binary pair as $\mu_{*} \sim 0.1 \mathrm{~J} / \mathrm{kg}$. This value can be used to estimate the typical speed of the inclusion's rise, $\mu_{*}^{1 / 2} U$. The typical non-dimensional value of the speed is $U \sim 0.1$, and thus the dimensional value of the inclusion's speed is ${ }_{540} \sim 1 \mathrm{~cm} / \mathrm{s}$. The correlation of this value with experimental measurements would allow an estimation of the parameter $\mu_{*}$ for a real binary mixture. The speed of the inclusion obtained here seems low, although it corresponds to the typical speeds of the so tiny inclusions (see e.g. [19]).

For the main calculations, the Grashof number was set as $\mathrm{Gr}=0.01$. Using its definition 10 , one concludes that the binary mixture is characterised by the relatively small density contrast, $\phi \sim 0.1$.

We may also correlate the coefficient of the surface tension to the real parameters. The dimensional value of the surface tension coefficient is given by $\sigma_{\text {dim }}=\rho_{*} \mu_{*} L_{*} \sigma$. If we accept that the non-dimensional value of the surface tension coefficient is $\sigma \sim 10^{-4}$, then the dimensional value can be estimated as $\sigma_{\text {dim }} \sim 10^{-5} \mathrm{~N} / \mathrm{m}$, which corresponds to the typical value of the surface tension at miscible interfaces [37.

For Pe $\sim 10^{8}$, the mobility coefficient can be estimated as, $\alpha \sim 10^{-6} \mathrm{~kg} \cdot \mathrm{s}$. $m^{-3}$. The bulk diffusion coefficient, far from the interfaces, can be calculated 555 as,

$$
D=\frac{\alpha \mu_{*}}{\rho_{*}}\left(\frac{\partial \mu_{0}}{\partial C}\right)=\frac{\alpha \mu_{*}}{\rho_{*}}\left[\frac{\frac{3}{4}}{\frac{1}{4}-C^{2}}-(3-2 \mathrm{~A})\right] .
$$

Hence the diffusion coefficient can be estimated as $D \sim 10^{-10} \mathrm{~m}^{2} \mathrm{~s}^{-1} 5$ Finally, the value of the capillary constant is $\epsilon \sim 10^{-13} \mathrm{~J} \cdot \mathrm{m}^{2} / \mathrm{kg}$.

Thus, the numerical results of this paper model the slow creeping rise of a tiny inclusion, with the density very close to the density of the ambient liquid, and with the low surface tension coefficient.

The above sequence could be helpful for understanding the desired values of non-dimensional parameters. For instance, a two-fold increase in the value of

\footnotetext{
${ }^{5}$ For estimation of the diffusion coefficient, the values of the concentration e.g. depicted in figure 5 may be used.
} 
the Reynolds number, with all other parameters kept at the same level, would roughly mean that the inclusion of the same size would rise twice faster, while the density contrast and the surface tension coefficient would be greater by four times. A two-fold increase in the Grashof number, with the other parameters kept unchanged, would mean that the binary mixture has twice larger density contrast. If the values of the non-dimensional parameters are kept the same, but the inclusion's initial size is twice larger, then the use of the above sequence would show that the speed of such an inclusion would be twice smaller due to the twice lower density contrast.

\section{Conclusions}

On the basis of the phase-field approach we model the rise of a Stokesian inclusion in a liquid, assuming that the inclusion is being slowly absorbed by the liquid. Frequently, the phase-field model is used as a numerical tool capable of tracing the complex transformations of immiscible phase boundaries [27, 28]. In our work, the phase-field approach is used as a physics-based model for the description of an evolution of a miscible heterogeneous binary mixture [43, 37. The main difference from the other works is that we take the initial thickness of an interface between the components of a mixture different from its equilibrium value. This change makes the intensity of the diffusion in the considered binary system much stronger, and as a result to reproduce the behaviour of an immiscible interface the values of the Peclet number should be taken considerably higher $\left(\left[10^{6} ; 10^{8}\right]\right.$ in the current work as compared with $\left[10^{2} ; 10^{4}\right]$ in 585 [27]). This difference makes the numerical modelling much more challenging, thus allowing us to model only the Stokesian rise of a tiny inclusion with the small density contrast. Nevertheless, the obtained results reveal a number of the new interesting effects.

The mathematical model for the binary mixture is characterised by the two time scales that define the rates of convective and diffusive changes. These scales are ultimately determined by the Reynolds and Peclet numbers, and the values 
of these parameters were chosen so to model the faster convective processes and the slower diffusive changes. Such a limit corresponds a more practical case of the slow absorption. In addition, in this limit we are able to draw some similarities with the immiscible case when the behaviour of a Stokesian inclusion is well understood.

Despite seemingly low dissolution rates considered, the effect of the absorption on the observed motion is enormous. The inclusions that are absorbed by the liquid rise faster than they would rise in a non-absorbing liquid. This effect is explained by the action of the Korteweg force, totally similar to the Marangoni force that affects the rise of the bubbles with surfactants [34, 35, 20, 36]. The absorption from the inclusion's surface occurs unevenly: the interfacial mass transfer is stronger at the top cap, where the gradient of the chemical potential is stronger. Owing to slow diffusion, the dissolved matter is not immediately absorbed by the ambient liquid, but accumulates at the inclusion's rare side forming the concentration wake. The rise of the inclusion in the closed domain is accompanied by the recirculation flow that is developed near the inclusion and that rises together with the inclusion.

The described complex dynamics of the mixing process of two slowly miscible fluids is essential for a better understanding of the chemical engineering processes. The reported results substantially extend the previous studies [29, 30, 31, 32], where the focus was on intensification of the weak dissolution from the bubbles, assuming that the reverse effect of diffusion on the bubble motion is negligible.

\section{Acknowledgments}

The financial support of the Royal Society (Ref. IE160277), and the Russian Foundation for Basic Research (Grant No. 16-5110079) is gratefully acknowledged. 


\section{References}

${ }_{620}$ [1] C. Perego, P. Ingallina, Recent advances in the industrial alkylation of aromatics: new catalysts ad new processes, Catalysis Today 73 (2002) 322 .

[2] H. Ganji, J. Ahari, A. Farshi, M. Kakavand, Modelling and simulation of benzene alkylation process reactors for production of ethylbenzene, Petroleum \& Coal 46 (2004) 55-63.

[3] K. A. Detrick, J. F. Himes, J. M. Meister, F.-M. Nowak, Uop hf alkylation technology, McGraw-Hill, 2004, Ch. 1.4, pp. 1.33-1.55.

[4] E. Khlebnikova, E. Ivashkina, I. Dolganova, Benzene alkylation with ethylene: The way to increase the process efficiency, Chemical Engineering and Processing: Process Intensification 120 (2017) 234-240.

[5] R. Dugas, Pilot plant study of carbon dioxide capture by aqueous monoethanolamine, Tech. rep., The University of Texas at Austine (May 2006).

[6] Y. Kim, J. Lim, S. Jeong, Y. Yoon, S. Bae, S. Nam, Comparison of carbon dioxide absorption in aqueous mea, dea, tea, and amp solutions, Bull. Korean Chem. Soc. 34 (2013) 783-787.

[7] R. Davies, F. Taylor, The mechanism of large bubbles rising through extended liquids and through liquids in tubes, Proceedings of the Royal Society A 200 (1950) 375.

[8] W. Haberman, R. Sayre, Motion of rigid and fluid spheres in stationary and moving liquids inside cylindrical tubes, Tech. Rep. Report 1143, David Taylor Model Basin (1958).

[9] D. Moore, The rise of a gas bubble in viscous liquid, J. Fluid Mech. 6 (1959) 113. 
[10] T. Taylor, A. Acrivos, On the deformation and drag of a falling viscous drop at low reynolds number, Journal of Fluid Mechanics 18 (1964) 466-476.

[11] R. Clift, J. Grace, M. Weber, Bubbles, Drops, and Particles, Academic Press, Inc., 1978.

[12] D. Bhaga, M. Weber, Bubbles in viscous liquids: shapes, wakes and velocities, J. Fluid Mech. 105 (1981) 61.

[13] G. Ryskin, L. Leal, Numerical simulation of free-boundary problems in fluid mechanics. part 2. buoyancy-driven motion of a gas bubble through a quiescent liquid, J. Fluid Mech. 148 (1984) 19.

[14] A. Blanco, J. Magnaudet, The structure of the high reynolds number flow around an ellipsoidal bubble of fixed shape, Phys. Fluids 7 (1995) 12651274.

[15] G. Bozzano, M. Dente, Shape and terminal velocity of single bubble motion: a novel approach, Computers \& Chemical Engineering 25 (2001) 571-576.

[16] A. Kulkarni, J. Joshi, Bubble formation and bubble rise velocity in gasliquid systems: A review, Ind. Eng. Chem. Res. 44 (2005) 5873-5931.

[17] G. Mougin, J. Magnaudet, Wake-induced forces and torques on a zigzagging/spiralling bubble, J. Fluid Mech. 567 (2006) 185.

[18] B. Figueroa-Espinoza, D. Legendre, Mass or heat transfer from spheroidal gas bubbles rising through a stationary liquid, Chemical Engineering Science 65 (2010) 6296-6309.

[19] D. Legendre, R. Zenit, R. Velez-Cordero, On the deformation of gas bubbles in liquids, Physics of Fluids 24 (2012) 043303.

[20] J. Magnaudet, I. Eames, The motion of high-reynolds-number bubbles in inhomogeneous flows, Annu. Rev. Fluid Mech. 32 (2000) 659-708. 
[29] F. Takemura, A. Yabe, Gas dissolution process of spherical rising gas bubbles, Chemical Engineering Science 53 (1998) 2691-2699.

[30] K. Tsuchiya, M. Koshihata, T. Tomida, T. Saito, Effect of satellite bubble on dynamics of gas absorption from a co2 bubble into a downward-flowing

[21] P. Ern, F. Risso, D. Fabre, J. Magnaudet, Wake-induced oscillatory paths of bodies freely rising of falling in fluids, Annu. Rev. Fluid Mech. 44 (2012) $97-121$.

[22] L. Chen, S. Garimella, J. Reizes, E. Leonardi, The development of a bubble rising in a viscous liquid, J. Fluid Mech. 387 (1999) 61.

[23] M. van Sint Annaland, N. Deen, J. Kuipers, Numerical simulation of gas bubbles behaviour using a three-dimensional volume of fluid method, Chem. Eng. Sci. 60 (2005) 2999.

[24] M. van Sint Annaland, W. Dijkhuizen, N. Deen, J. Kuipers, Numerical simulation of behavior of gas bubbles using a 3-d front-tracking method, AIChE Journal 52 (2006) 99-110.

[25] J. Hua, J. Lou, Numerical simulation of bubble rising in viscous liquid, J. Comput. Phys. 222 (2007) 769-795.

[26] J. Hua, J. Stene, P. Lin, Numerical simulation of 3d bubbles rising in viscous liquids using a front tracking method, J. Comput. Phys. 227 (2008)

[27] H. Ding, P. Spelt, C. Shu, Diffuse interface model for incompressible twophase flows with large density ratios, Journal of Computational Physics 226 (2007) 2078-2095.

[28] M. Tripathi, K. Sahu, R. Govindarajan, Why a falling drop does not in general behave like a rising bubble, Scientific Reports 4:4771 (2014) 1-8. liquid, Korean Journal of Chemical Engineering 16 (1999) 635-639. 
[31] K. Tsuchiya, T. Saito, T. Kajishima, S. Kosugi, Coupling between mass transfer from dissolving bubbles and formation of bubble-surface wave, Chemical Engineering Science 56 (2001) 6411-6417.

[32] A. Mohamed, M. Herrada, J. Lopez-Herrera, A. N. Ganan-Calvo, Isothermal dissolution of small rising bubbles in a low viscosity liquid, Chemical Engineering and Processing: Process Intensification 6 (2014) 113.

[33] F. Ozkan, A. Wenka, E. Hansjosten, P. Pfeifer, B. Kraushaar-Czarnetzki, Numerical investigation of interfacial mass transfer in two phase flows using the vof method, Engineering Applications of Computational Fluid Mechanics 10 (2016) 100-110.

[34] V. Levich, Physicochemical hydrodynamics, Englewood Cliffs, N.J. : Prentice-Hall, 1962.

[35] C. Ybert, J.-M. di Meglio, Ascending air bubbles in protein solutions, The European Physical Journal B 4 (1998) 313-319.

[36] S. Tasoglu, U. Demirci, M. Muradoglu, The effect of soluble surfactant on the transient motion of a buoyancy-driven bubble, Physics of fluids 20 (2008) 040805.

[37] A. Vorobev, Dissolution dynamics of miscible liquid/liquid interfaces, Current Opinion in Colloid and Interface Science 19 (2014) 300-308.

[38] J. Cahn, J. Hilliard, Free energy of a nonuniform system. i. interfacial free energy, Journal of Chemical Physics 28 (1958) 258-267.

[39] R. Xie, A. Vorobev, On the phase-field modelling of a miscible liquid/liquid boundary, J. Colloid Interface Sci 464 (2016) 48-58.

[40] A. Vorobev, A. Boghi, Phase-field modelling of a miscible system in spinning droplet tensiometer, J. Colloid Interface Sci 482 (2016) 193-204.

[41] P. Flory, Principles of polymer chemistry, Cornell University Press, 1953. 
[42] J. Lowengrub, L. Truskinovsky, Quasiincompressible cahnhilliard fluids and topological transitions, Proceedings of the Royal Society A 454 (1998) $2617-2654$.

[43] A. Vorobev, Boussinesq approximation of the cahn-hilliard-navier-stokes equations, Phys. Rev. E 82 (2010) 056312.

[44] A. Kheniene, A. Vorobev, Linear stability analysis of a horizontal phase boundary separating two miscible liquids, Phys. Rev. E 88 (2013) 022404.

[45] A. Kheniene, A. Vorobev, Linear stability of a horizontal phase boundary subjected to shear motion, European Physical Journal E 38 (2015) 77.

[46] A. Vorobev, A. Ivantsov, T. Lyubimova, Phase-field modelling of gravitycapillary waves on a miscible interface, European Physical Journal E 40.

[47] L. Landau, E. Lifshitz, Fluid Mechanics (Volume 6 of Course of Theoretical Physics), Elsevier. Butterworth-Heinemann, 1987. 